\title{
On the infinitely-stable rotational mechanism using the off-axis rotation of a bistable translational mechanism
}

\author{
G. Hao and J. Mullins \\ School of Engineering-Electrical and Electronic Engineering, University College Cork, Cork, Ireland \\ Correspondence to: G. Hao (g.hao@ucc.ie)
}

Received: 4 December 2014 - Revised: 24 April 2015 - Accepted: 23 May 2015 - Published: 5 June 2015

\begin{abstract}
Different from the prior art concentrating on the primary translation of bistable translational mechanisms this paper investigates the off-axis rotation behaviour of a bistable translational mechanism through displacing the guided primary translation at different positions. Moment-rotation curves obtained using the nonlinear finite element analysis (FEA) for a case study show the multiple stable positions of the rotation under each specific primary motion, suggesting that an infinitely-stable rotational mechanism can be achieved by controlling the primary motion. In addition, several critical transition points have been identified and qualitative testing has been conducted for the case study.
\end{abstract}

\section{Introduction}

A compliant mechanism is multistable if it has more than two stable translational/rotational positions that refer to zero force/moment points with positive stiffness (Oh, 2008; Howell et al., 2013). Multistable compliant mechanisms have a variety of successful applications such as switches, valves, relays, grasper, adaptive end effectors, sensors, energy harvesting devices and vibration isolators (Oh, 2008; Howell et al., 2013; Lassooij et al., 2012; Chen and Lan, 2012; Hansen et al., 2007; Liu et al., 2013; Shaw et al., 2013). Unlike the traditional ways of using locking mechanisms and detents, a compliant mechanism based multistable mechanism obtains multistability through the storage and release of potential energy stored in their flexible members during post-buckling.

This paper focuses on bistable translational mechanisms using fixed-clamped beams (Lassooij et al., 2012; Chen and Lan, 2012; Hansen et al., 2007; Dunning et al., 2012; Kim and Ebenstein, 2012; Holst et al., 2011; Zhang and Chen, 2013), rather than using the fixed-pinned (Qiu et al., 2004) and the pinned-pinned beams (Sonmez and Tutum, 2008) that are hard to manufacture monolithically. There are mainly two methods to design the fixed-clamped bistable translational mechanism (Dunning et al., 2012). One method is to use the fixed-guided beam via an inclined arrangement where the beam has no deformation at the initial position without the input force. The other method is to pre-stress the fixed ends of a pair of fixed-clamped non-inclined beams to introducing buckling where the beam deforms at the initial position without the input force. However, the second method is out of scope of this paper. The bistable translational mechanism studied in this paper is shown in Fig. 1a, which is composed of two fixed-clamped inclined beams connected at the middle (motion end). Under the primary force only, each beam will work like a fixed-guided beam due to the symmetrical nature. The typical force-displacement relation of this bistable mechanism in the primary direction is shown in Fig. 1c with the critical points and regions being marked.

Different from the above reported works in Lassooij et al. (2012), Chen and Lan (2012), Hansen et al. (2007), Dunning et al. (2012), Kim and Ebenstein (2012), Holst et al. (2011) and Zhang and Chen (2013) which employ the primary translation to achieve finite multi-stable statuses, this paper studies on the multistable off-axis rotation behaviour of the bistable translational mechanism in order to obtain an infinitely-stable rotational mechanism. This off-axis rotation behavior with multistable points has also potential applications in human joint rehabilitative devices, dynamic and static balancing of machines, and human mobility-assisting devices (Hou and Lan, 2013) when combining with a positive stiffness spring. 


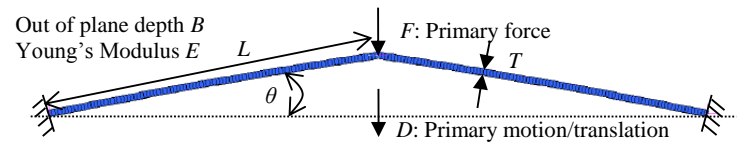

(a) A simplified CAD model in Strand7 for the bistable mechanism

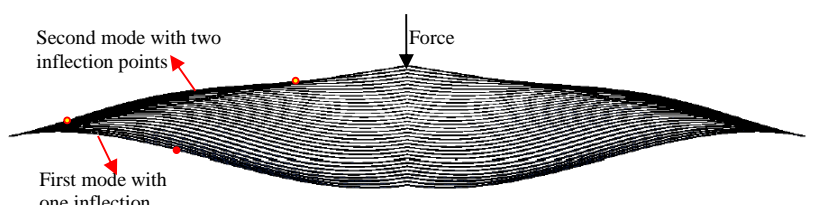

inflection

(b) Beam shape results of the bistable mechanism obtained from Strand7 during post buckling: One inflection point (leftmost one) disappears during snap-through

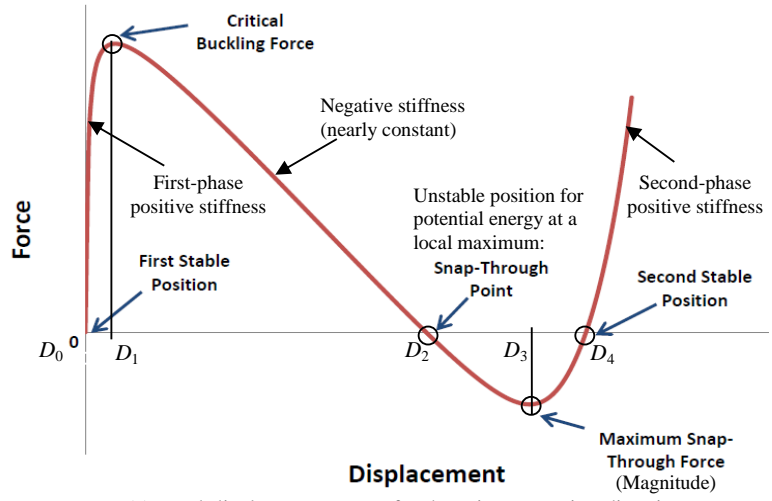

(c) Load-displacement curve for the primary motion direction

Figure 1. Embodiment of the bistable mechanism.

This paper is organized as follows. Section 2 describes the displacement-controlled FEA (finite element analysis) simulation. The off-axis rotational analysis of a bistable translational mechanism is implemented in Sect. 3 showing the infinite stable rotational positions. A conceptual design of an infinitely-stable rotational mechanism is followed. Discussions are drawn in Sect. 4.

\section{Displacement-controlled FEA simulation}

Due to the strongly nonlinear characteristics of post-buckling behaviour, it is very difficult to model buckled beams correctly. There have been a large amount of works on accurately modelling the force-displacement characteristics of buckled beams as well as modelling the exact curvature and inflection points of the beam as it travels between two stable equilibrium points. These modelling techniques include the curve decomposition method (Kim and Ebenstein, 2012) and elliptic or comprehensive elliptic integral solutions (Holst et al., 2011; Zhang and Chen, 2013). Each has provided an insight into the post-buckling behaviour of compliant mechanisms, but has its own limitations. Compared to the method using the comprehensive elliptic integral in Zhang and Chen (2013), the combined method in Holst et al. (2011) or the curve decomposition method in Kim and Ebenstein (2012) is unable to solve large deflections of thin beams with multiple inflection points and subject to any kinds of load cases. The models in Kim and Ebenstein (2012) and Zhang and Chen (2013) ignoring axial elongation can also causes that the linear region that the beam undergoes before the buckling point disappears and that the snap-through region is also incorrect, while the combined model (Holst et al., 2011) incorporating the axial elongation is much closer to the actual experimental result.

Nonlinear FEA (Dunning et al., 2012), however, can accurately predict post-buckling behaviour of bistable mechanisms as well as accurate stress values without any assumptions as mentioned above, which surpasses these fast numerical methods in Kim and Ebenstein (2012), Holst et al. (2011) and Zhang and Chen (2013). In addition, nonlinear FEA can analyse off-axis characteristics that these methods in Kim and Ebenstein (2012), Holst et al. (2011) and Zhang and Chen (2013) cannot deal with. It can be easily employed by most engineers and researchers.

As the force-displacement curve for post-buckling beams goes through a negative stiffness range, simple forcecontrolled simulations are not suitable since the controlled force is no longer a monotonic control parameter. Therefore, the bistable mechanism analysis needs displacementcontrolled FEA simulation. For the simulation cases in bistable mechanisms, there is a necessity to apply an enforced displacement and measure the load generated at the restrained node. Enforced-displacement control is what is needed to correctly model the post-buckling behaviour of a beam, especially if the off-axis analysis for example is being tested. In the off-axis analysis, the beam must be fixed (preloaded) at a specified position while other loads/displacements are applied, which cannot be achieved using force-controlled methods.

The nonlinear FEA in this paper was performed in Strand7 software, a powerful nonlinear FEA solver, using the 20node brick meshing element. The customizability of Strand7 allows the modelling of the bistable mechanism to be easily achieved. The simulation method is to first apply an enforced displacement and then to determine the resultant reaction forces developed due to these enforced displacements. The simulations can be modified to simultaneously change restraints and loads. The Strand7 solver is also much more intuitive allowing the user to monitor the solution convergence behaviour with a convergence graph when a simulation is running for example. The FEA solver can be monitored and adjusted in much greater detail.

\section{Off-axis rotational analysis}

In this section, a case study is analysed for investigating the off-axis rotation behaviour. The beam length was set up to $L=50 \mathrm{~mm}$ with the in-plane thickness $T=1 \mathrm{~mm}$, out-ofplane depth $B=5 \mathrm{~mm}$, inclined angle $\theta=10^{\circ}$ and Young's Modulus $E=2.4 \mathrm{GPa}$. In order to avoid the high-order buck- 


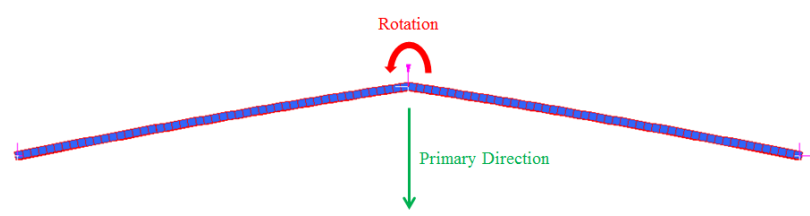

(a) Initial configuration

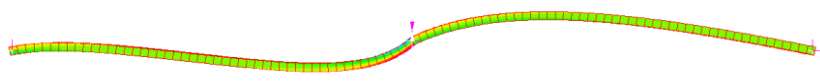

(b) Buckled beam being rotated

Figure 2. Off-axis rotation.

ling with high peek force and high stress, an optimised slight curvature $\left(1 / 1000 \mathrm{~mm}^{-1}\right)$ (Dunning et al., 2012) was applied to the beam, which is trivial in comparison to the beam length but is enough to perturb the beam to buckle at lower bending modes (Fig. 1b).

The FEA simulations of in-plane off-axis rotation for the bistable mechanism (Fig. 2) were performed on Strand7. Before implementing the off-axis rotational analysis, the primary translational analysis of the bistable mechanism was conducted as shown in Fig. 3. As can be seen approximately, $D_{1}=1.1 \mathrm{~mm}, D_{2}=9.1 \mathrm{~mm}, D_{3}=11.7 \mathrm{~mm}$ and $D_{4}=13.2 \mathrm{~mm}$ when referring to Fig. 1c.

To determine the off-axis rotational behaviour, the bistable mechanism was guided (by applied constraint) to displace only in the primary motion direction ( $y$ axis) by the displacement-controlled FEA simulation. For each guided primary displacement increment, the rotational test was performed where the bistable mechanism was displaced in the rotation direction and the reaction moment in this rotational direction was then recorded. Under the conditions that the primary motion is incremented from 0 to $14 \mathrm{~mm}$ with $0.5 \mathrm{~mm}$ each step and that the rotation is incremented from 0 to $15^{\circ}$ with $0.15^{\circ}$ each step, the graph of moment versus rotation is shown in Fig. 4. The stable rotational points can be observed from the FEA results when there is a zero moment point with a positive rotational stiffness. Similarly, the instable rotational points can be seen when there is a zero moment point with a negative rotational stiffness.

It is shown from Fig. 4a and b that there is no other stable rotational point except the zero point (home position) for $Y=0 \mathrm{~mm}$ or $Y>13.0 \mathrm{~mm}$, which complies with our expectation. For $Y=0$ the bistable mechanism also acts as a conventional compliant rotational joint with positive stiffness thereby having only one stable rotational point at the home position. For $Y>13.0 \mathrm{~mm}$ the bistable mechanism is approximately beyond the second stable point of the primary translation with positive stiffness, so it is expected to have only positive rotational stiffness over the rotation range (i.e. only one zero stable rotational point). For each primary motion $Y$ over $[0.5 \mathrm{~mm}, 12.5 \mathrm{~mm}$, there are either three or

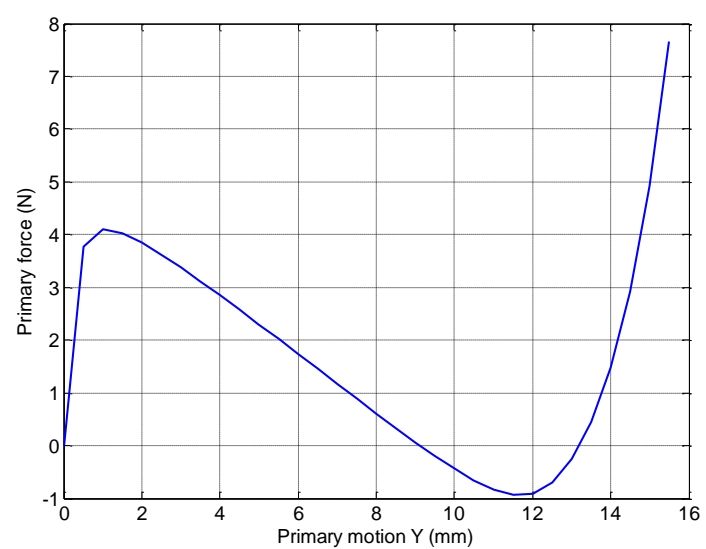

Figure 3. Load-displacement relation in the primary direction for the case study.

two stable points of the rotation. Figure 5 shows the number of the stable rotational points with regard to each primary motion, and indicates that after about $Y=3.0 \mathrm{~mm}$ but before $Y=12.5 \mathrm{~mm}$, the number of stable points reduces from three (two non-zero positions and one zero position) to two (two non-zero positions). The positive stable rotational position corresponding to each primary motion is shown in Fig. 6. It can be found that the stable position increases before a critical transition point at about $Y=6.0 \mathrm{~mm}$ but decreases after this critical point. It can be also noted from Fig. 4 that near the zero rotational point the rotational stiffness is approximately zero under small specific primary motion (except $Y=0$ ) or under the specific primary motion between $Y=12.5 \mathrm{~mm}$ and $Y=13.0 \mathrm{~mm}$, i.e. there is approximately constant moment.

It is interesting to be seen that with the increase of the primary motion $Y$, the positive rotational displacement at the maximal negative moment point increases before $Y=3.0 \mathrm{~mm}$ but decreases after $Y=3.0 \mathrm{~mm}$, and the maximal negative moment value has an increasing trends before $Y=7.0 \mathrm{~mm}$ but a decreasing trends after $Y=7.0 \mathrm{~mm}$.

Therefore, by controlling the primary motion from $Y=3.5$ to $12.5 \mathrm{~mm}$ as shown in Figs. 5 and 6, the mechanism can automatically rotate to one stable rotational status for any primary motion since the rotation zero point is an unstable position. This suggests that an infinitely-stable mechanism can be achieved due to the fact that there are infinite stable positions of rotation corresponding to infinite primary motion positions. A conceptual design of the infinitely-stable rotational mechanism is shown in Fig. 7.

In order to qualitatively verify the multistable off-axis rotation behaviour as discussed in this section, a similar (simple) prototype made of polycarbonate was fabricated by $\mathrm{CNC}$ milling machining as shown in Fig. 8. By manually displacing the bistable mechanism in the primary translational direction using the sliding guide, the rotational bistable behaviors at two specific primary translation positions $(Y=6.0$ and 
(a)

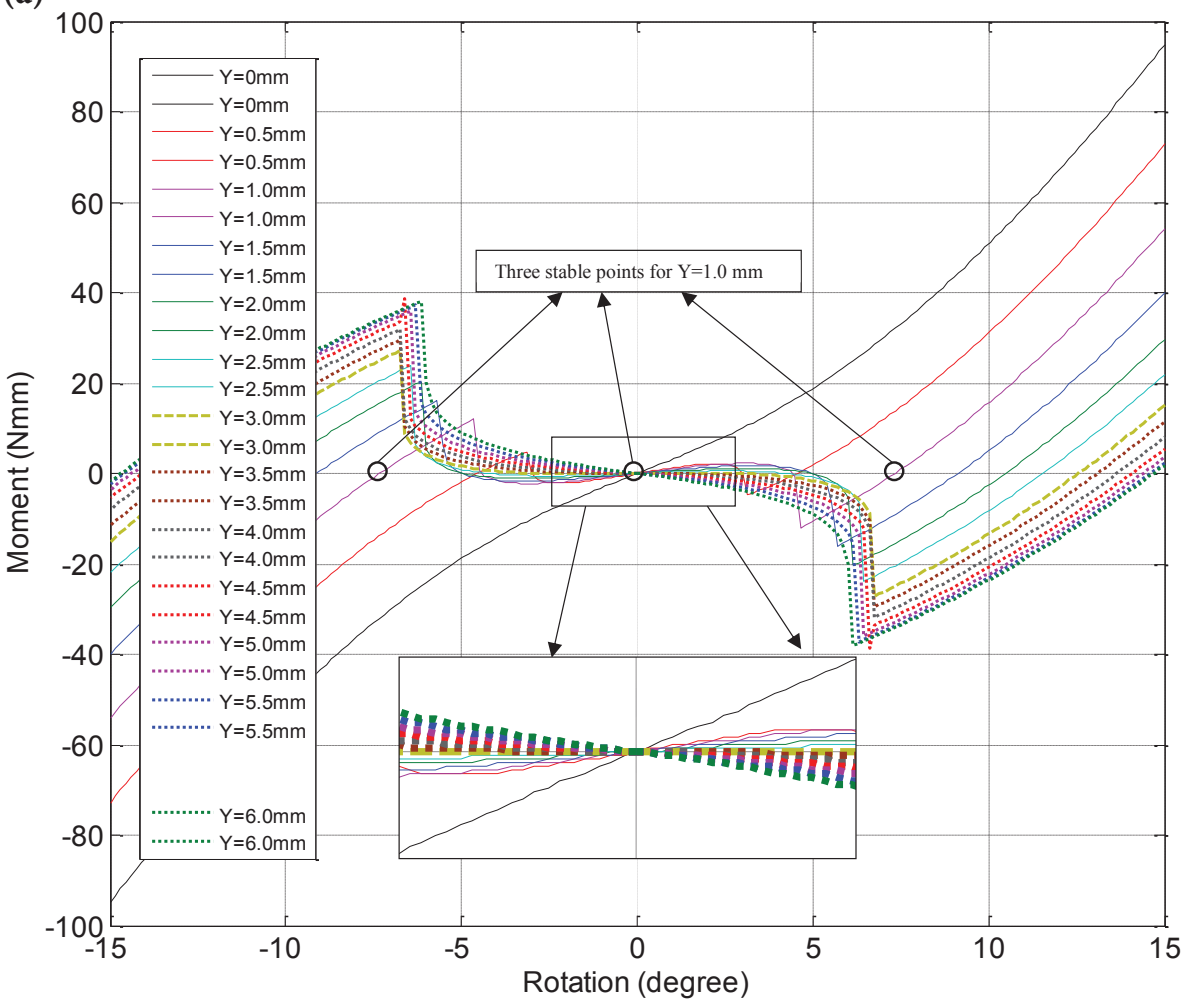

(b)

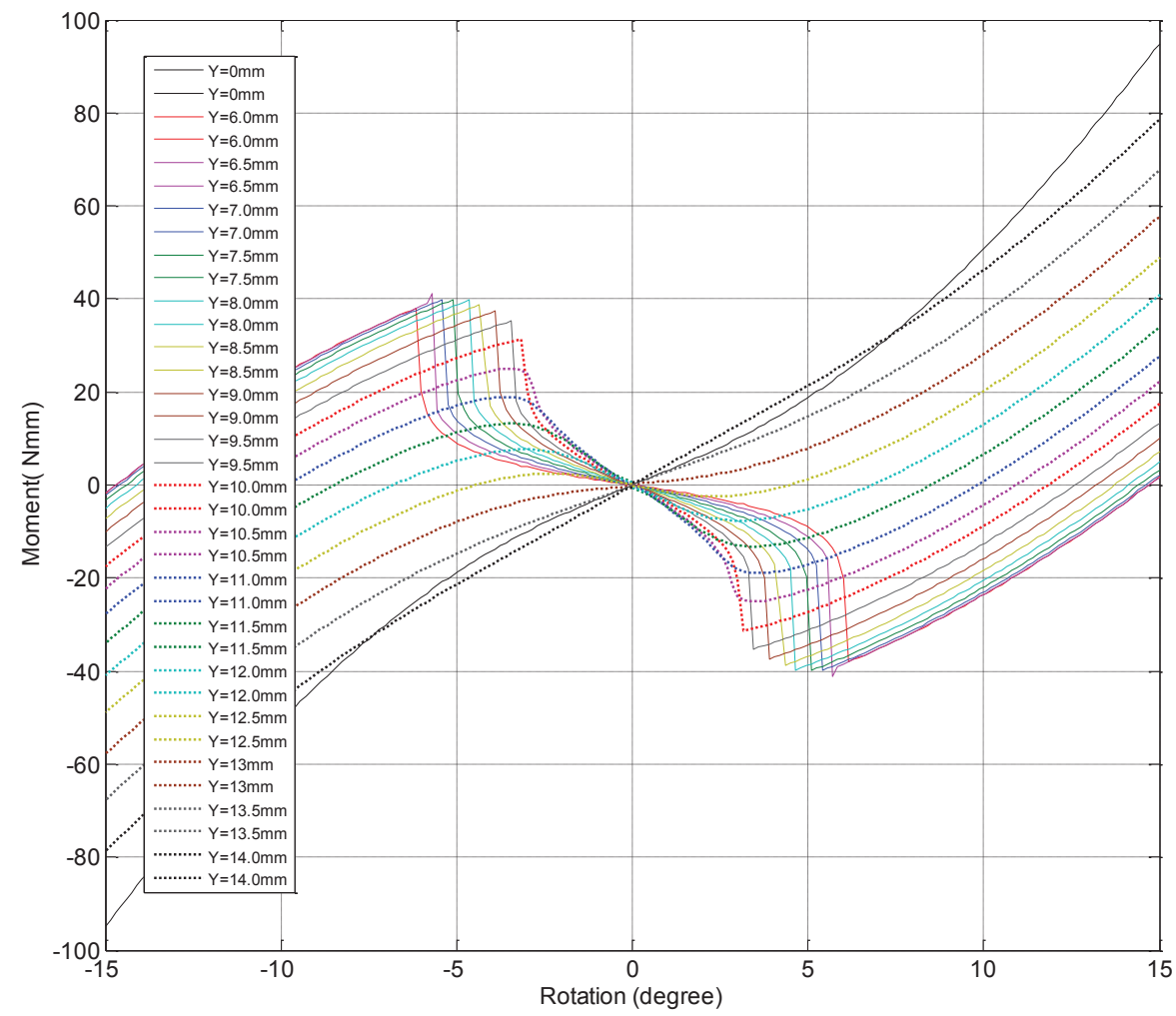

Figure 4. Moment versus rotation graph for the bistable mechanism. 


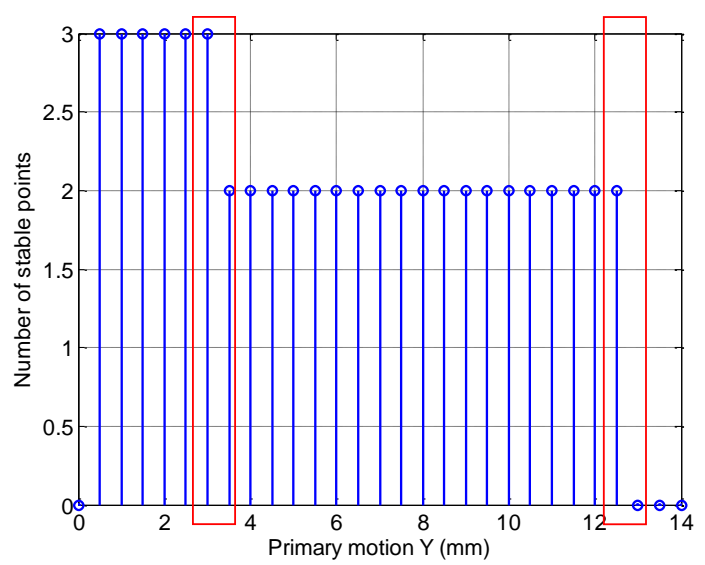

Figure 5. Number of stable rotational points under the specified primary motion.

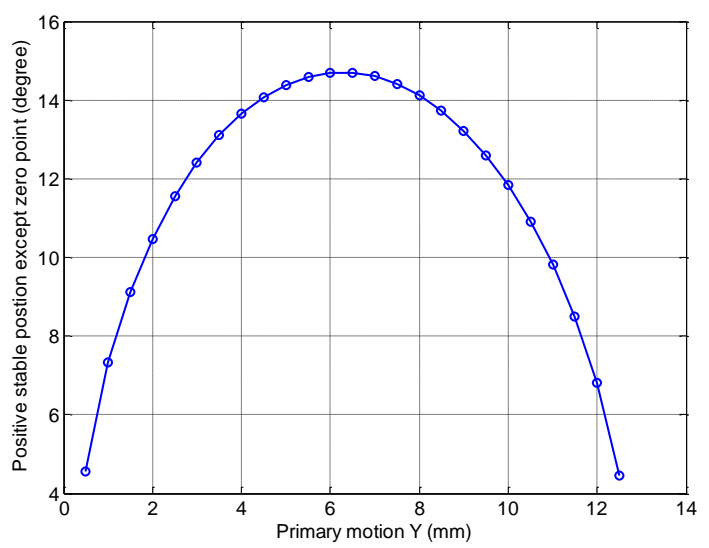

Figure 6. Positive stable rotational positions under different primary motions.

$10.5 \mathrm{~mm}$ ) have been observed (Fig. 8c-f). It is shown that the stable rotational displacement is larger under $Y=6.0 \mathrm{~mm}$ than that under $Y=10.5 \mathrm{~mm}$, which agrees with the FEA resutls (Fig. 6).

\section{Conclusions}

The off-axis rotation characteristics of a bistable translational mechanism have been investigated in detail through displacing the guided primary translation/motion at different positions. For each primary motion, a moment-rotation curve has been obtained using the nonlinear FEA, which shows the multistable statues of the rotation. The idea of an infinitelystable rotational mechanism that can be stable at any rotation position is proposed by controlling the primary motion.

Future works include fast numerical modelling, experimental testing verification and geometrical optimization for the off-axis rotation behavior.

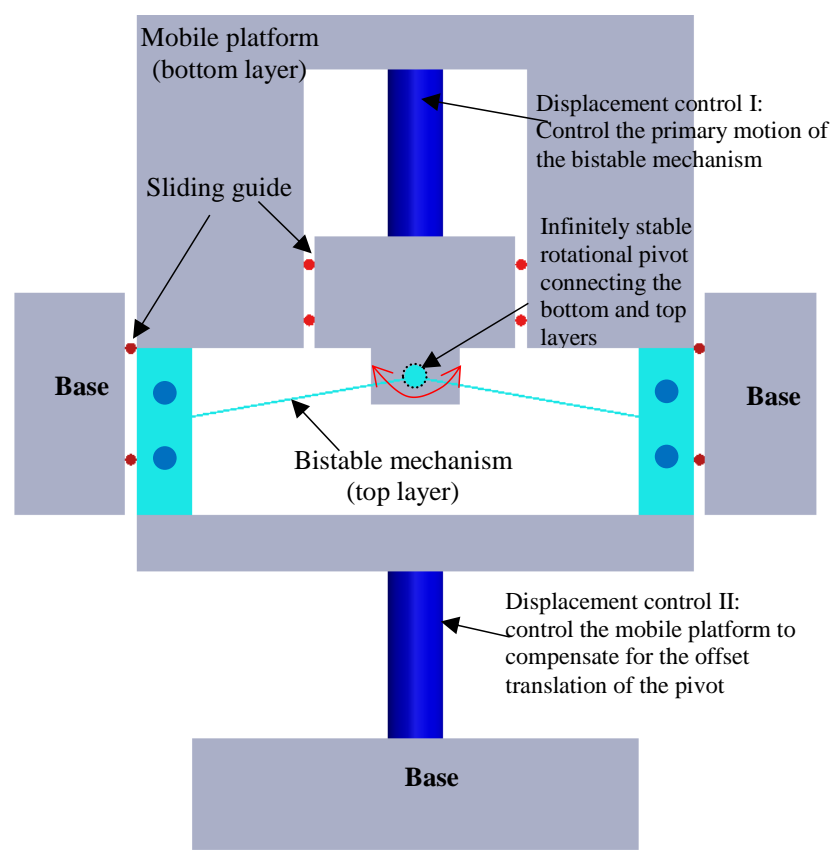

Figure 7. Schematic deisgn for the infinitely-stable rotational mechanism.

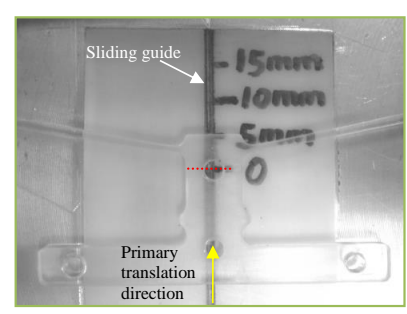

(a) First stable position of the primary motion

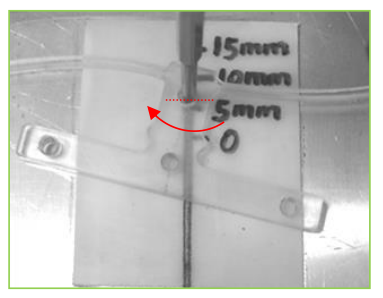

(c) First stable rotational position under $\mathrm{Y}=6.0 \mathrm{~mm}$

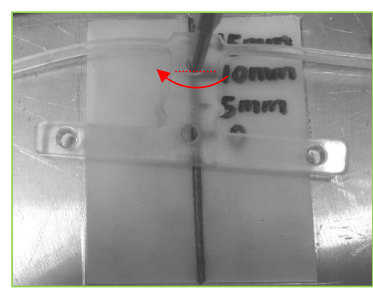

(e) First stable rotational position under $\mathrm{Y}=10.5 \mathrm{~mm}$

Figure 8. Fabricated prototype.
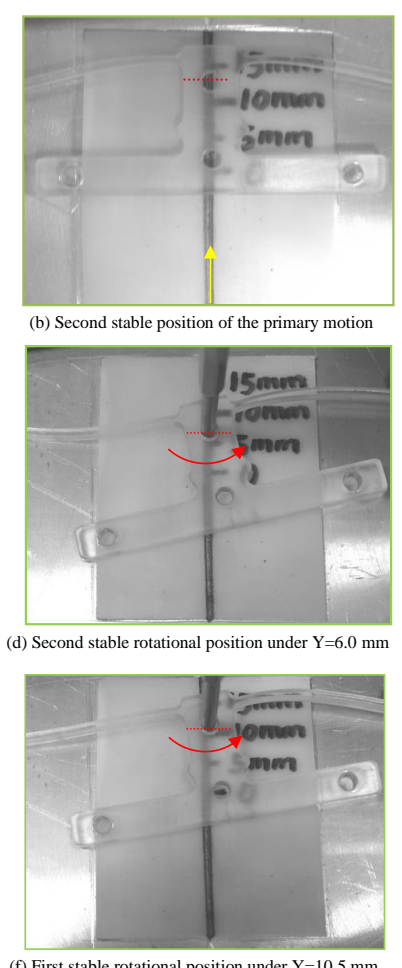

(f) First stable rotational position under $\mathrm{Y}=10.5 \mathrm{~mm}$ 
Acknowledgements. Tim Power and Michael O'Shea in University College Cork are greatly appreciated for fabricating the prototype.

Edited by: J. Schmiedeler

Reviewed by: two anonymous referees

\section{References}

Chen, Y. H. and Lan, C. C.: An Adjustable Constant-Force Mechanism for Adaptive End-Effector Operations, J. Mech. Design, 134, 031005, doi:10.1115/1.4005865, 2012.

Dunning, A. G., Tolou, N., and Pluimers, P. P.: Bistable Compliant Mechanisms: Corrected Finite Element Modeling for Stiffness Tuning and Preloading Incorporation, J. Mech. Design, 134, 084502, doi:10.1115/1.4006961, 2012.

Hansen, B. J., Carron, C. J., and Hawkins, A. R.: Plastic Latching Accelerometer Based on Bistable Compliant Mechanisms, Smart Mater. Struct., 16, 1967-1972, 2007.

Holst, G. L., Teichert, G. H., and Jensen, B. D.: Modeling and Experiments of Buckling Modes and Deflection of FixedGuided Beams in Compliant Mechanisms, J. Mech. Design, 133, 051002, doi:10.1115/1.4003922, 2011.

Hou, C.-W. and Lan, C.-C.: Functional Joint Mechanisms with Constant-torque Outputs, Mech. Mach. Theory, 62, 166-181, 2013.

Howell, L. L., Magleby, S. P., and Olsen, B. M.: Handbook of Compliant Mechanisms, Wiley, New York, 2013.
Kim, C. and Ebenstein, D.: Curve Decomposition for Large Deflection Analysis of Fixed-Guided Beams for Statically Balanced Compliant Mechanisms, J. Mech. Robot., 4, 041009, doi:10.1115/1.4007488, 2012.

Lassooij, J., Tolou, N., Tortora, G., Caccavaro, S., Menciassi, A., and Herder, J. L.: A Statically Balanced and Bi-stable Compliant End Effector Combined with a Laparoscopic 2-DoF Robotic Arm, Mech. Sci., 3, 85-93, 2012.

Liu, W. Q., Badel1, A., Formosa1, F., Wu, Y. P., and Agbossou, A.: Novel Piezoelectric Bistable Oscillator Architecture for Wideband Vibration Energy Harvesting, Smart Mater. Struct., 22, 035013, doi:10.1088/0964-1726/22/3/035013, 2013.

Oh, Y.: Synthesis of Multistable Equilibrium Compliant Mechanisms, PhD thesis, University of Michigan, Michigan, USA, 2008.

Qiu, J., Lang, J., and Slocum, A.: A Curved-Beam Bistable Mechanism, J. Microelectromech. Syst., 13, 137-146, 2004.

Shaw, A. D., Neild, S. A., Wagg, D. J., Weaver, P. M., and Carrella, A.: A Nonlinear Spring Mechanism Incorporating a Bistable Composite Plate for Vibration Isolation, J. Sound Vibrat., 33, 6265-6275, 2013.

Sonmez, U. and Tutum, C. C.: A Compliant Bistable Mechanism Design Incorporating Elastica Buckling Beam Theory and Pseudo-Rigid-Body Model, J. Mech. Design, 130, 042304, doi:10.1115/1.2839009, 2008.

Zhang, A. and Chen, G.: A Comprehensive Elliptic Integral Solution to the Large Deflection Problems of Thin Beams in Compliant Mechanisms, J. Mech. Robot., 5, 021006, doi:10.1115/1.4023558, 2013. 